\section{Flat gastric epithelial neoplasm detected by endo- scopic screening with autofluorescence imaging video endoscopy}

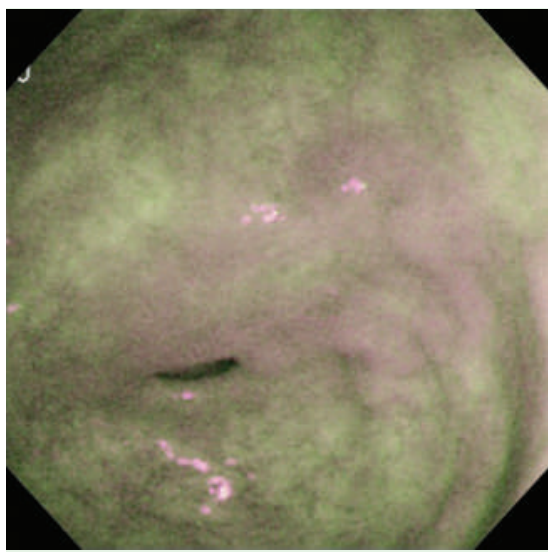

Fig. 1 Autofluorescence imaging (AFI) showed a blurred purple area in a patient with a history of endoscopic submucosal resection for early gastric cancer.

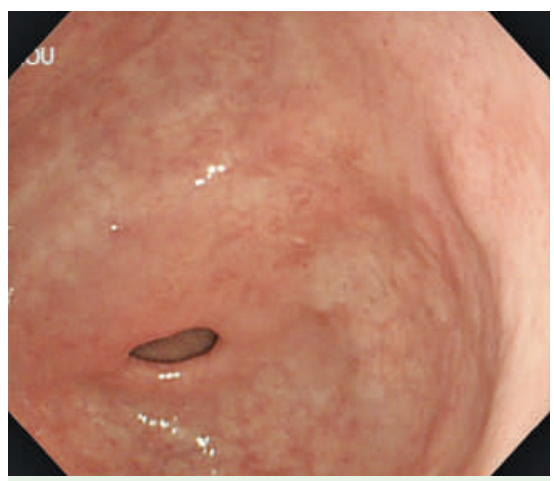

Fig. 2 In the white-light image, neither the extent or even the presence of the lesion was indicated.

Autofluorescence imaging (AFI) video endoscopy provides real-time color images from the computerization of captured fluorescence emitted from natural endogenous fluorophores that have been excited by light of specific wavelength. With early gastric cancers, AFI can visualize flat tumors, or the extent of isochromatic tumors, in a way that white-light endoscopy cannot [1]. Because of the difference in fluorescence properties between neoplasia and normal tissue, it was able to reveal a flat neoplasm that did not show up under white-light endoscopy.

A 79-year-old man with history of endoscopic submucosal dissection for early gastric cancer was scheduled to undergo follow-up examination in our endoscopy

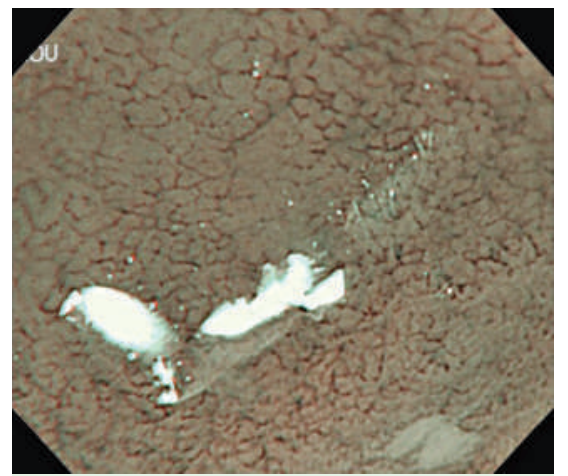

Fig. 3 Magnifying narrow-band imaging (NBI) showed the fine-network microvascular pattern in the center of the tumor.

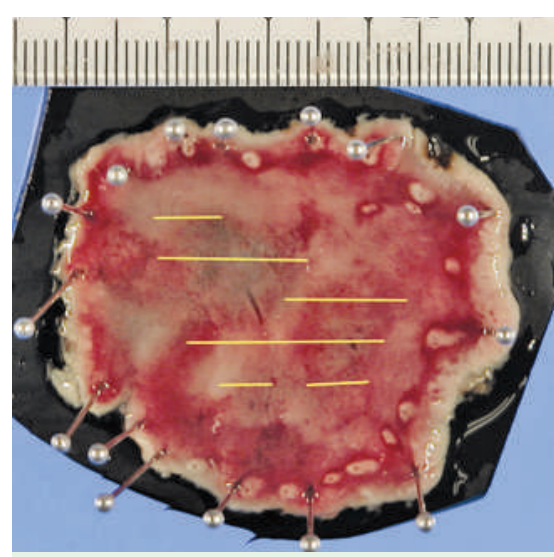

Fig. 4 Histological mapping of the resected specimen: the intramucosal neoplasm (yellow lines) had been removed en bloc.

\section{Video 1}

Autofluorescence imaging (AFI) found blurred purple areas in the prepylorus, whereas the lesion was not apparent in white-light images. Magnifying narrow-band imaging (NBI) then revealed a fine-network vascular pattern that indicated well-differentiated adenocarcinoma.

unit. Endoscopic screening was done using an AFI protosystem.

The system consists of an image processor (XCV-260HP; Olympus Medical Systems Corp., Tokyo, Japan), a light source (XCLV-260HP), and a dedicated video endoscope (XGIF-Q240FZ). The system works in white light, AFI, or narrow-band imaging (NBI) modes; the mode is changed by pressing a small button on a control head. The scope has a zoom function with a maximum of $\times 80$. The AFI function can be used to detect suspicious lesions and then magnifying NBI can provide detailed inspection of the detected lesions [2]. A black rubber attachment (MB-162; Olympus) was fitted to the tip of the endoscope.

In our patient, autofluorescence observation showed a $20-\mathrm{mm}$ blurred purple area in the prepylorus ( $\bullet$ Fig. 1) that was not clear in white-light images ( $\bullet$ Fig. 2). Magnifying NBI showed a fine-network microvascular pattern in the center, suggesting a differentiated-type adenocarcinoma ( Fig. 3) [3]. With the extent of the tumor being established by both AFI and magnifying NBI, it was removed by endoscopic submucosal dissection. Histological examination of the resected specimen showed that it was well-differentiated tubular adenocarcinoma confined to the mucosa and with clear margins (๑ Fig. 4, Video 1).

Endoscopy_UCTN_Code_CCL_1AB_2AD_3AB

\section{K. Tatsumi ${ }^{1}$, N. Uedo ${ }^{1}$, R. Ishihara ${ }^{1}$,} H. lishi ${ }^{1}$, M. Matsumura ${ }^{2}$, S. Ishiguro ${ }^{2}$

1 Department of Gastrointestinal Oncology, Osaka Medical Center for Cancer and Cardiovascular Diseases, Osaka, Japan

2 Department of Pathology, Osaka Medical Center for Cancer and Cardiovascular Diseases, Osaka, Japan

\section{References}

1 Uedo $N$, Iishi H, Tatsuta $M$ et al. A novel videoendoscopy system by using autofluorescence and reflectance imaging for diagnosis of esophagogastric cancers. Gastrointest Endosc 2005; 62: $521-528$

2 Kara MA, Peters FP, Fockens $P$ et al. Endoscopic video-autofluorescence imaging followed by narrow band imaging for detecting early neoplasia in Barrett's esophagus. Gastrointest Endosc 2006; 64: 176-185

3 Nakayoshi T, Tajiri H, Matsuda K et al. Magnifying endoscopy combined with narrowband imaging system for early gastric cancer: correlation of vascular pattern with histopathology [including video]. Endoscopy 2004 ; $36: 1080-1084$

Bibliography

DOI $10.1055 / \mathrm{s}-2007-966727$

Endoscopy 2007; 39: E289

(c) Georg Thieme Verlag KG Stuttgart · New York . ISSN 0013-726X

\section{Corresponding author}

\section{N. Uedo, MD}

Department of Gastrointestinal Oncology Osaka Medical Center for Cancer and Cardiovascular Diseases, 3-3 Nakamichi 1-chome, Higashinari-ku, Osaka 537-8511, Japan

Fax: +81-6-69814067

uedou-no@mc.pref.osaka.jp 